\title{
The Use of Curl-Conforming Basis Functions for the Magnetic-Field Integral Equation
}

\author{
Özgür Ergül, Student Member, IEEE, and Levent Gürel, Senior Member, IEEE
}

\begin{abstract}
Divergence-conforming Rao-Wilton-Glisson (RWG) functions are commonly used in integral-equation formulations to model the surface current distributions on planar triangulations. In this paper, a novel implementation of the magnetic-field integral equation (MFIE) employing the curl-conforming $\hat{\boldsymbol{n}} \times \mathbf{R W G}$ basis and testing functions is introduced for improved current modelling. Implementation details are outlined in the contexts of the method of moments, the fast multipole method, and the multilevel fast multipole algorithm. Based on the examples of electromagnetic modelling of conducting scatterers, it is demonstrated that significant improvement in the accuracy of the MFIE can be obtained by using the curl-conforming $\hat{\boldsymbol{n}} \times$ RWG functions.
\end{abstract}

Index Terms-Fast multipole method, integral equations, magnetic-field integral equation (MFIE), method of moments (MoM).

\section{INTRODUCTION}

The magnetic-field integral equation (MFIE) [1]-[4] is widely used for electromagnetic modelling of three-dimensional conductor problems with closed surfaces. The MFIE formulation is usually followed by a discretization in order to render the problem amenable to solution with a numerical technique, such as the method of moments (MOM) [5], the fast multipole method (FMM) [6], [7], or the multilevel fast multipole algorithm (MLFMA) [7], [8]. A popular choice for discretization is to triangulate the surface of the geometry and employ the Rao-Wilton-Glisson (RWG) functions [9] defined on the planar triangular subdomains (Fig. 1) as basis functions (BFs) to expand the unknown induced surface current. Despite the widespread success of similar discretizations of the electric-field integral equation (EFIE), MFIE ${ }^{1}$ implementations using the RWG functions are reported to be plagued with accuracy problems [10], [11], i.e., for the same discretization, MFIE results are significantly less accurate compared to EFIE results, especially when the geometry of the problem includes sharp wedges and corners. It is demonstrated in Section II that the accuracy problem arises even for relatively smooth geometries, such as a sphere. Similar remarks are also valid for

Manuscript received January 27, 2005; revised June 25, 2005. This work was supported in part by the Turkish Academy of Sciences in the framework of the Young Scientist Award Program (LG/TUBA-GEBIP/2002-1-12), in part by the Scientific and Technical Research Council of Turkey (TUBITAK) under Research Grant 105E172, and in part by contracts from ASELSAN and SSM.

The authors are with the Department of Electrical and Electronics Engineering, Bilkent University, TR-06800, Bilkent, Ankara, Turkey (e-mail: ergul@ee.bilkent.edu.tr; lgurel@bilkent.edu.tr).

Digital Object Identifier 10.1109/TAP.2006.877159

${ }^{1}$ Even for the solution of the same physical problem, EFIE and MFIE operators are different, and therefore, the same basis functions may not work the same way for both. the combined-field integral equation (CFIE) [2] that contains both the MFIE and the EFIE as two components. Careful investigations [11] indicate that neither the numerical evaluation of the singular MFIE integrals [12], nor the incorrect use of the solid-angle factor [13], [14], is the fundamental cause of this accuracy problem. This finding stimulates further studies, especially for a better understanding of how the discretization of the MFIE and the modelling of the surface current affects the accuracy of the results [15]. Therefore, it becomes desirable to employ other types of BFs defined on triangular domains in addition to the RWG functions.

In this paper, we present efficient and reliable schemes for the numerical discretization of the MFIE with the curl-conforming $\hat{\boldsymbol{n}} \times$ RWG [16]-[21] basis and testing functions (BTFs) defined on planar triangulations. Two different formulations to calculate the elements of the impedance matrix will be given in detail. In the first formulation, the relations between the elements of the impedance matrix obtained by using the $\hat{\boldsymbol{n}} \times \mathrm{RWG}$ and the RWG functions with the Galerkin method will be also established. The second formulation, which also has a counterpart for the RWG functions, will be presented as an alternative and more efficient way to calculate the interactions. Furthermore, the Appendix will outline the factorization of the interactions for the FMM implementations employing the $\hat{\boldsymbol{n}} \times$ RWG BTFs, similar to the FMM formulations involving the RWG functions [7]. Finally, we will compare the results obtained by using the $\hat{\boldsymbol{n}} \times$ RWG and the RWG functions, and demonstrate the improvement in the accuracy of the solutions due to the $\hat{\boldsymbol{n}} \times$ RWG functions.

\section{PRos AND CONS OF THE MFIE}

In this section, we will point to the disadvantages of using the MFIE (either alone or in a CFIE setting) as well as its advantages. We will emphasize its accuracy problem as one of the most significant among other shortcomings of the MFIE. Finally, we will argue that the advantages of using the MFIE within a CFIE setting are so significant that the MFIE remains indispensable despite its disadvantages.

\section{A. Advantages of the MFIE}

Since the MFIE is formulated as a second-kind integral equation, its conditioning properties are superior to those of the EFIE, which is a first-kind integral equation. This is of major importance especially for iterative solutions, i.e., the MFIE usually converges faster than the EFIE. Furthermore, the MFIE does not have the low-frequency breakdown problem that is exhibited by the EFIE. MFIE is also more suitable for hybridization with the physical optics (PO) method since both are based on the same magnetic-field boundary condition. 


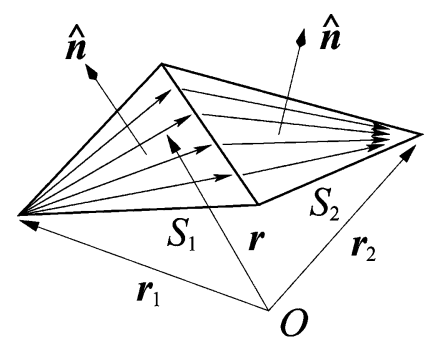

(a)

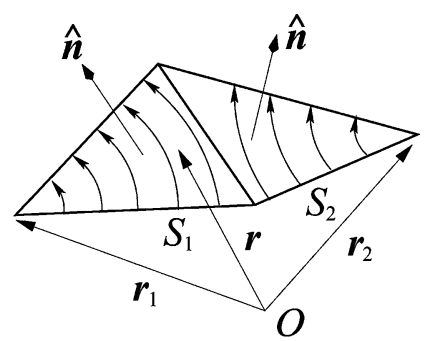

(b)
Fig. 1. Spatial distributions of (a) RWG and (b) $\hat{n} \times$ RWG functions.

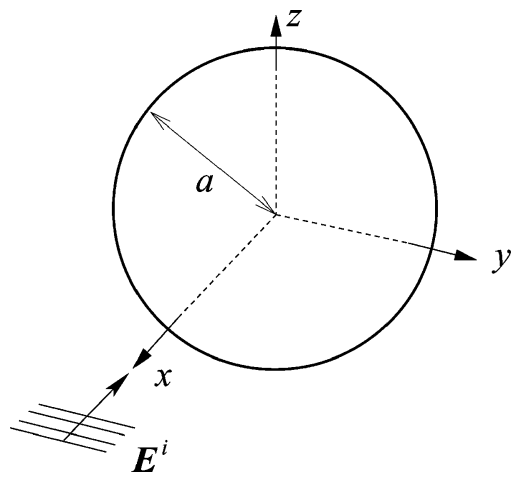

Fig. 2. Scattering from a perfectly conducting sphere of radius $a$.

\section{B. Disadvantages of the MFIE}

The restriction of the MFIE's applicability to only closed surfaces is its greatest shortcoming. Similar to the EFIE, the MFIE also has a nonempty null space that results in the interior-resonance problem in the vicinities of discrete frequencies. Unlike the EFIE, the MFIE produces nonsymmetric matrix equations, even for Galerkin implementations. Another disadvantage of the MFIE is its poor accuracy, which will be demonstrated in the next subsection.

\section{Accuracy of the MFIE}

Despite the many advantages of the MFIE, it suffers from an accuracy problem [11]. In order to demonstrate that the inherent inaccuracy of the MFIE is not relieved even by refining the mesh at the expense of increasing the number of unknowns, we consider the scattering problem depicted in Fig. 2. A conducting sphere with radius $a$ is illuminated by a plane wave at two different frequencies corresponding to $a=\lambda$ and $a=2 \lambda$. In addition to obtaining the analytical solution with the Mie series, the scattering problem is also solved with the EFIE, the MFIE, and the CFIE. For this purpose, the surface of the sphere is discretized with various triangulations of coarse to fine densities, corresponding to 1000 to 30,000 unknowns, respectively.

Figs. 3 and 4 present the backscattered and forward-scattered RCS values, respectively, obtained with various mesh densities. The dots in the plots correspond to the $\lambda / 10$ triangulation density at each frequency. Clearly, the EFIE results readily converge to the reference analytical results for mesh densities even smaller than $\lambda / 10$, whereas the MFIE results manifest significant discrepancies with the analytical results not only for $\lambda / 10$ triangulation, but also for much higher mesh densities. We confirm that similar accuracy problems are observed in the MFIE

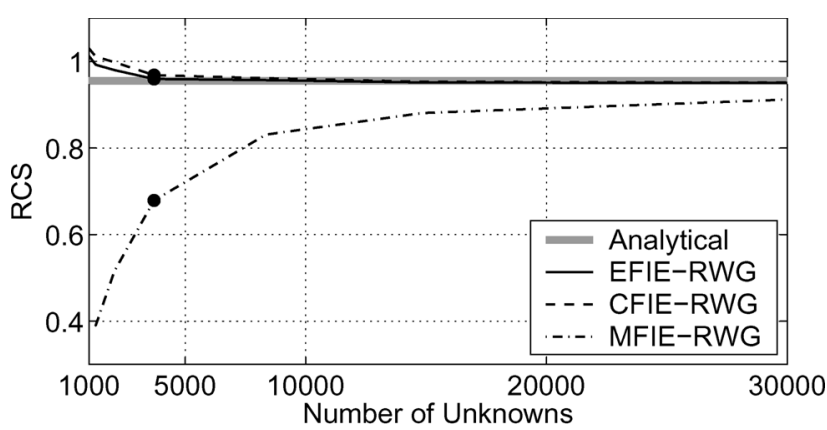

(a)

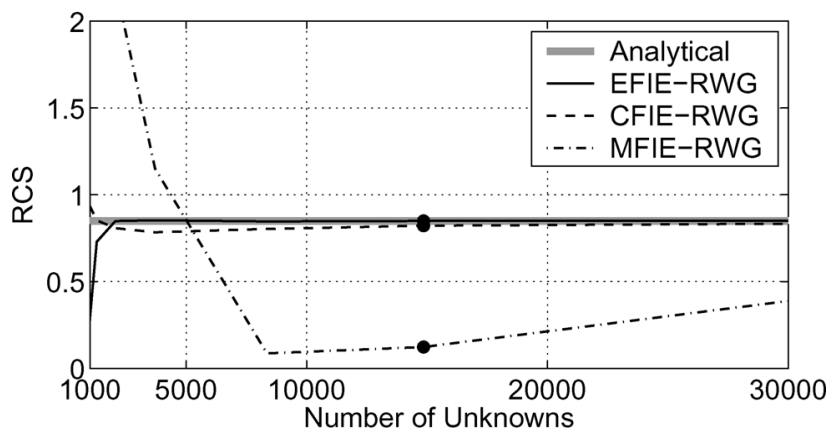

(b)

Fig. 3. Normalized backscattered RCS (RCS $/ \pi a^{2}$, dimensionless) of the perfectly conducting sphere of radius (a) $a=\lambda$ and (b) $a=2 \lambda$ with respect to the number of unknowns. The dots on the curves correspond to the $\lambda / 10$ discretization.

solutions of various other geometries, such as a cube that has distinctly different features than a sphere due to its sharp edges and corners.

The CFIE solution is obtained by combining the EFIE and MFIE with multipliers 0.2 and 0.8 , respectively. That the CFIE solutions are contaminated with the MFIE inaccuracy according to the $0.2 \div 0.8$ ratio is clearly visible in Fig. 4 . Even though the backscattering RCS values presented in Fig. 3 do not display the same level of inaccuracy for CFIE solutions, it should be emphasized that the backscattering and forward-scattering RCS values in Figs. 3 and 4 are obtained from the same CFIE solutions. Therefore, based on the comparison of the Mie Series, EFIE, MFIE, and CFIE solutions presented in Fig. 4, we conclude that the CFIE solutions bear the inaccuracies caused by the MFIE.

\section{Advantages of the CFIE}

Although the CFIE is merely a linear combination of the EFIE and the MFIE [2], it is remarkable that the CFIE has better conditioning properties than both the EFIE and the MFIE [22]. Hence, the need to accelerate the iterative solutions necessitates the use of the CFIE. Furthermore, the CFIE eliminates the interior-resonance problems due to both the EFIE and the MFIE. Therefore, the CFIE is an essential asset for fast iterative solvers, such as the FMM and the MLFMA, which, in turn, makes the MFIE indispensable, too. Even though the inaccuracy problems of the MFIE affects the accuracy of the CFIE results adversely, abandoning the MFIE in favor of the EFIE cannot be an option since a convergent solution may not be available in some cases. Then, we should keep the MFIE and devise ways to improve the 


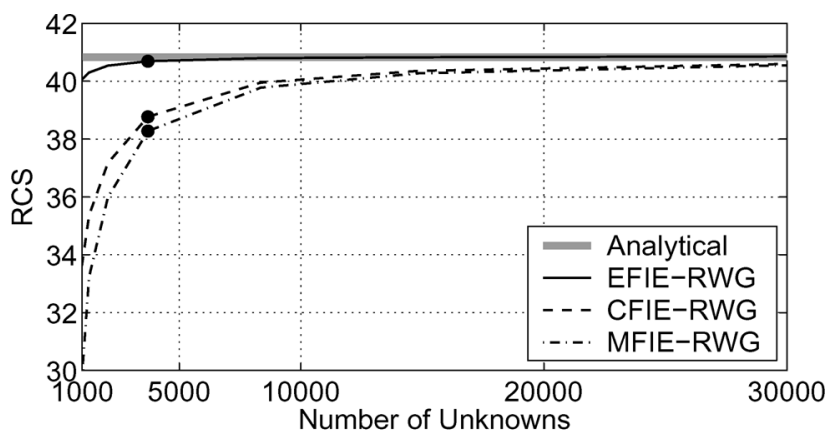

(a)

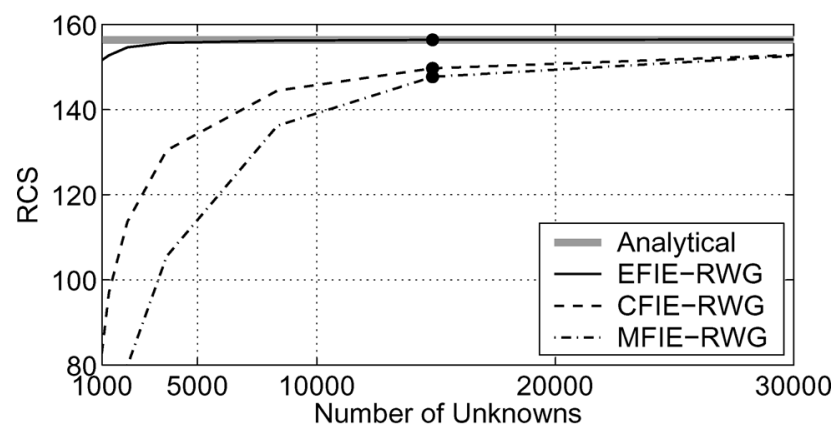

(b)

Fig. 4. Normalized forward-scattered RCS (RCS $/ \pi a^{2}$, dimensionless) of the perfectly conducting sphere of radius (a) $a=\lambda$ and (b) $a=2 \lambda$ with respect to the number of unknowns. The dots on the curves correspond to the $\lambda / 10$ discretization.

accuracy of the MFIE solutions in order to obtain efficient and accurate CFIE solutions.

\section{DISCRETIZATION OF THE MFIE}

For conducting scatterers with closed surfaces, the MFIE can be formulated by using the boundary condition for the tangential magnetic field on the surface as

$$
\boldsymbol{J}(\boldsymbol{r})-\hat{\boldsymbol{n}} \times \int_{S} d \boldsymbol{r}^{\prime} \boldsymbol{J}\left(\boldsymbol{r}^{\prime}\right) \times \nabla^{\prime} g\left(\boldsymbol{r}, \boldsymbol{r}^{\prime}\right)=\hat{\boldsymbol{n}} \times \boldsymbol{H}^{\boldsymbol{i}}(\boldsymbol{r})
$$

where the scattered magnetic field is expressed in terms of the induced (unknown) surface current $\boldsymbol{J}(\boldsymbol{r})$. In (1), the observation point $\boldsymbol{r}$ approaches the surface from the outside, $\hat{\boldsymbol{n}}$ is the outwardly directed normal, $\boldsymbol{H}^{\boldsymbol{i}}(\boldsymbol{r})$ is the incident magnetic field, and

$$
g\left(\boldsymbol{r}, \boldsymbol{r}^{\prime}\right)=\frac{e^{i k R}}{4 \pi R} \quad\left(R=\left|\boldsymbol{r}-\boldsymbol{r}^{\prime}\right|\right)
$$

denotes the free-space Green's function in phasor notation with the $e^{-i w t}$ convention. To discretize the MFIE in (1), $\boldsymbol{J}(\boldsymbol{r})$ is expanded in a series of BFs $\boldsymbol{b}_{n}(\boldsymbol{r})$ as

$$
\boldsymbol{J}(\boldsymbol{r})=\sum_{n=1}^{N} a_{n} \boldsymbol{b}_{n}(\boldsymbol{r})
$$

where $a_{n}$ is the unknown coefficient of the $n$th BF. Then, the boundary condition is tested by using the inner product of the magnetic field and testing functions (TFs) $\boldsymbol{t}_{m}(\boldsymbol{r})$ so that an $N \times$ $N$ matrix equation is obtained as

$$
\sum_{n=1}^{N} Z_{m n} a_{n}=v_{m}, \quad m=1, \ldots, N
$$

where $Z_{m n}$ is the matrix element derived as

$$
\begin{aligned}
Z_{m n}=\int_{S_{m}} d \boldsymbol{r} \boldsymbol{t}_{m}(\boldsymbol{r}) \cdot \boldsymbol{b}_{n}(\boldsymbol{r}) \\
\quad-\int_{S_{m}} d \boldsymbol{r} \boldsymbol{t}_{m}(\boldsymbol{r}) \cdot \hat{\boldsymbol{n}} \times \int_{S_{n}} d \boldsymbol{r}^{\prime} \boldsymbol{b}_{n}\left(\boldsymbol{r}^{\prime}\right) \times \nabla^{\prime} g\left(\boldsymbol{r}, \boldsymbol{r}^{\prime}\right)
\end{aligned}
$$

and $v_{m}$ is the element of the excitation vector derived as

$$
v_{m}=\int_{S_{m}} d \boldsymbol{r} \boldsymbol{t}_{m}(\boldsymbol{r}) \cdot \hat{\boldsymbol{n}} \times \boldsymbol{H}^{i}(\boldsymbol{r}) .
$$

In (5) and (6), $S_{n}$ and $S_{m}$ represent the surfaces, on which the $n$th basis and the $m$ th testing functions are defined, respectively. Edge-based BTFs are usually defined on a pair of surfaces sharing a common edge, e.g., both the RWG and the $\hat{\boldsymbol{n}} \times$ RWG BTFs can be expressed as

$$
\begin{aligned}
\boldsymbol{b}_{n}(\boldsymbol{r}) & =\boldsymbol{b}_{n, 1}(\boldsymbol{r})+\boldsymbol{b}_{n, 2}(\boldsymbol{r}) \\
\boldsymbol{t}_{m}(\boldsymbol{r}) & =\boldsymbol{t}_{m, 1}(\boldsymbol{r})+\boldsymbol{t}_{m, 2}(\boldsymbol{r})
\end{aligned}
$$

where the "half functions" on individual triangles are decomposed, i.e., the second subscript denotes either one of the pair of neighboring triangles sharing a common edge with index $n$ or $m$. Then, the interaction between the half BF $\boldsymbol{b}_{n, j}$ and the half TF $\boldsymbol{t}_{m, i}$ for $i=1,2$ and $j=1,2$ can be written as

$$
\begin{aligned}
Z_{m n, i j} & =\int_{S_{m, i}} d \boldsymbol{r} \boldsymbol{t}_{m, i}(\boldsymbol{r}) \cdot \boldsymbol{b}_{n, j}(\boldsymbol{r}) \\
& -\int_{S_{m, i}} d \boldsymbol{r} \boldsymbol{t}_{m, i}(\boldsymbol{r}) \cdot \hat{\boldsymbol{n}} \times \int_{S_{n, j}} d \boldsymbol{r}^{\prime} \boldsymbol{b}_{n, j}\left(\boldsymbol{r}^{\prime}\right) \times \nabla^{\prime} g\left(\boldsymbol{r}, \boldsymbol{r}^{\prime}\right) .
\end{aligned}
$$

The formulation in (1) and the expressions for the elements of the impedance matrix in (5) and (8) are appropriate for both the divergence-conforming and the curl-conforming BTFs since the differential operator is placed on the Green's function. In addition to this "first formulation," there are two other formulations with the differential operator residing on either the $\mathrm{BF}$ or the TF [12]. The "second formulation" in [12] is applicable to curl-conforming BFs with no restriction on the TF, whereas the "third formulation" in [12] places no restriction on the BF while requiring the TF to be divergence-conforming. Therefore, it is possible to use curl-conforming basis and/or testing functions with a proper choice of the available MFIE formulations. 
In this paper, we will choose both the basis and the testing functions as the curl-conforming $\hat{\boldsymbol{n}} \times \mathrm{RWG}$ functions within the context of the "first formulation" represented by (5) and (8). This "first formulation" is also widely used in the MOM implementations of the MFIE for the RWG functions [12], [23]. For sake of brevity, details of computing the singular integrals, singularity extraction, and evaluation of analytical integrals therein [12], [13], [23]-[25] will not be included in this paper.

\section{A. Comment on the Choice of BFs}

The main idea of a paper [26] published by our group in 1999 was that BFs used for current modelling should also be capable of properly modelling the inherent charge distribution. We proved that the RWG BFs satisfied that requirement unconditionally. Appraised by the same criterion, the $\hat{\boldsymbol{n}} \times \mathrm{RWG}$ functions would have failed since they are not divergence-conforming and would not model the charge distribution correctly. However, as we will show in the present paper, $\hat{\boldsymbol{n}} \times$ RWG functions model the current distribution even better than the RWG functions, when used to discretize the MFIE. This apparent contradiction needs an explanation. We note that the previous study [26] considered only the EFIE, but not the MFIE. The hypersingular term of the EFIE is customarily evaluated by distributing the double differential operators onto the BTFs. Then, the divergences of the BTFs become explicitly necessary in the EFIE formulation. Moreover, divergence of the BF is even physically interpreted as the charge distribution owing to the continuity equation

$$
\nabla \cdot \boldsymbol{J}(\boldsymbol{r})=i w \rho(\boldsymbol{r})
$$

If an attempt was made to use the $\hat{\boldsymbol{n}} \times$ RWG functions, which are not divergence conforming, as the BFs in such an EFIE implementation, their divergences would create singular "blade" functions on the edges of the entire triangulation that would mathematically undermine the computations and that could physically be interpreted as artificial line-charge accumulation and gaps in the conducting surface. Consequently, an attempt of employing the $\hat{\boldsymbol{n}} \times$ RWG BFs in the EFIE would have failed. On the other hand, the three MFIE formulations considered in [12] and in present paper do not explicitly require the divergence of the BF, i.e., neither of those MFIE formulations demands the $\mathrm{BF}$ to be divergence-conforming.

Following the line of thinking in the above, it remains to be investigated whether the hypersingular terms of the EFIE can be evaluated differently, i.e., by not placing one of the differential operator on the BF, and whether the EFIE will accept a curlconforming BF under such a modification. This question will be addressed elsewhere.

\section{B. Matrix Elements With $\hat{\boldsymbol{n}} \times R W G$ BTFs}

The $\hat{\boldsymbol{n}} \times$ RWG functions have the spatial distribution as shown in Fig. 1(b), which can be expressed as

$$
\boldsymbol{f}(\boldsymbol{r})=\hat{\boldsymbol{n}} \times \boldsymbol{f}^{R}(\boldsymbol{r})
$$

where $\boldsymbol{f}^{R}(\boldsymbol{r})$ is the spatial distribution of the associated RWG function shown in Fig. 1(a) and defined as [9]

$$
\boldsymbol{f}^{R}(\boldsymbol{r})=\left\{\begin{array}{cr}
\frac{l}{2 A_{1}}\left(\boldsymbol{r}-\boldsymbol{r}_{1}\right), & r \in S_{1} \\
\frac{l}{2 A_{2}}\left(\boldsymbol{r}_{2}-\boldsymbol{r}\right), & r \in S_{2} \\
0 & \text { otherwise }
\end{array}\right\} .
$$

In (11), $A_{1}$ and $A_{2}$ are the areas of the surfaces $S_{1}$ and $S_{2}$, respectively, while $l$ is the length of the common edge shared by the triangles. Inserting the definition of the $\hat{\boldsymbol{n}} \times$ RWG functions into (8), we can obtain the expression for the interaction between the half $\hat{\boldsymbol{n}} \times$ RWG functions as

$$
\begin{aligned}
Z_{m n, i j}^{\mathrm{nRWG}}=\int_{S_{m, i}} d \boldsymbol{r} \boldsymbol{t}_{m, i}^{R}(\boldsymbol{r}) \cdot \boldsymbol{b}_{n, j}^{R}(\boldsymbol{r})-\int_{S_{m, i}} d \boldsymbol{r} \boldsymbol{t}_{m, i}^{R}(\boldsymbol{r}) \\
\quad \cdot \int_{S_{n, j}} d \boldsymbol{r}^{\prime}\left[\hat{\boldsymbol{n}}^{\prime} \times \boldsymbol{b}_{n, j}^{R}\left(\boldsymbol{r}^{\prime}\right)\right] \times \nabla^{\prime} g\left(\boldsymbol{r}, \boldsymbol{r}^{\prime}\right)
\end{aligned}
$$

where $\boldsymbol{b}_{n, j}^{R}(\boldsymbol{r})$ and $\boldsymbol{t}_{m, i}^{R}(\boldsymbol{r})$ are the half of the RWG functions associated with the $j$ th triangle of the $n$th edge and $i$ th triangle of the $m$ th edge, respectively. We note that (12) reduces to

$$
Z_{m n, i j}^{\mathrm{nRWG}}=\frac{1}{2} \int_{S_{m, i}} d \boldsymbol{r} t_{m, i}^{R}(\boldsymbol{r}) \cdot \boldsymbol{b}_{n, j}^{R}(\boldsymbol{r})
$$

when the testing and the basis triangles are the same, i.e., in the case of the self interaction of a triangle.

A comparison of (12) to (8) shows that the first term obtained by using the $\hat{\boldsymbol{n}} \times$ RWG functions is the same as the corresponding term that would be obtained by using the RWG functions. In other words, the diagonal elements of the impedance matrices are the same for the implementations of the $\hat{\boldsymbol{n}} \times \mathrm{RWG}$ and the RWG functions:

$$
Z_{m m}^{\mathrm{RWG}}=Z_{m m}^{\mathrm{nRWG}} \text {. }
$$

Furthermore, (12) can be rewritten as

$$
\begin{aligned}
Z_{m n, i j}^{\mathrm{nRWG}}=\int_{S_{m, i}} d \boldsymbol{r} \boldsymbol{t}_{m, i}^{R}(\boldsymbol{r}) & \cdot \boldsymbol{b}_{n, j}^{R}(\boldsymbol{r})+\int_{S_{n, j}} d \boldsymbol{r}^{\prime} \boldsymbol{b}_{n, j}^{R}\left(\boldsymbol{r}^{\prime}\right) \cdot \hat{\boldsymbol{n}}^{\prime} \\
& \times \int_{S_{m, i}} d \boldsymbol{r} \boldsymbol{t}_{m, i}^{R}(\boldsymbol{r}) \times \nabla g\left(\boldsymbol{r}, \boldsymbol{r}^{\prime}\right)
\end{aligned}
$$

by interchanging the order of the integrals in the second term. Then, comparison of the second terms in (8) and (15) indicates that

$$
Z_{m n}^{\mathrm{nRWG}}=-Z_{n m}^{\mathrm{RWG}}
$$

when the functions (defined on pairs of triangles) related to edges $m$ and $n$ do not overlap in space. It should be noted that a 
combination of (14) and (16) should be used for those nondiagonal elements of the impedance matrices involving interactions between the BTFs overlapping on a triangle. Hence, we observe that all interactions calculated for the $\hat{\boldsymbol{n}} \times$ RWG and the RWG functions are closely related.

\section{AltERNATIVE FORMULATION}

To derive an alternative formulation for the $\hat{\boldsymbol{n}} \times$ RWG functions, (12) is manipulated as

$$
\begin{aligned}
Z_{m n, i j}^{\mathrm{nRWG}}= & \int_{S_{m, i}} d \boldsymbol{r} \boldsymbol{t}_{m, i}^{R}(\boldsymbol{r}) \cdot \boldsymbol{b}_{n, j}^{R}(\boldsymbol{r}) \\
& -\int_{S_{m, i}} d \boldsymbol{r} \boldsymbol{t}_{m, i}^{R}(\boldsymbol{r}) \cdot \int_{S_{n, j}} d \boldsymbol{r}^{\prime} \boldsymbol{b}_{n, j}^{R}\left(\boldsymbol{r}^{\prime}\right) \frac{\partial g\left(\boldsymbol{r}, \boldsymbol{r}^{\prime}\right)}{\partial n^{\prime}} \\
& +\int_{S_{m, i}} d \boldsymbol{r} \boldsymbol{t}_{m, i}^{R}(\boldsymbol{r}) \cdot \hat{\boldsymbol{n}}^{\prime} \int_{S_{n, j}} d \boldsymbol{r}^{\prime} \boldsymbol{b}_{n, j}^{R}\left(\boldsymbol{r}^{\prime}\right) \cdot \nabla^{\prime} g\left(\boldsymbol{r}, \boldsymbol{r}^{\prime}\right)
\end{aligned}
$$

where we use

$$
\hat{\boldsymbol{n}}^{\prime} \cdot \nabla^{\prime} g\left(\boldsymbol{r}, \boldsymbol{r}^{\prime}\right)=\frac{\partial g\left(\boldsymbol{r}, \boldsymbol{r}^{\prime}\right)}{\partial n^{\prime}}
$$

and take the normal vector $\hat{\boldsymbol{n}}^{\prime}$ in the third term outside the inner integral since it is constant over the basis triangle. For this term, we apply the identity for the scalar $s$ and the vector $\boldsymbol{v}$ as

$$
\nabla \cdot(s \boldsymbol{v})=\nabla s \cdot \boldsymbol{v}+s \nabla \cdot \boldsymbol{v}
$$

and change the placement of the differential operator to obtain

$$
\begin{aligned}
Z_{m n, i j}^{\mathrm{nRWG}}= & \int_{S_{m, i}} d \boldsymbol{r} \boldsymbol{t}_{m, i}^{R}(\boldsymbol{r}) \cdot \boldsymbol{b}_{n, j}^{R}(\boldsymbol{r}) \\
& -\int_{S_{m, i}} d \boldsymbol{r} \boldsymbol{t}_{m, i}^{R}(\boldsymbol{r}) \cdot \int_{S_{n, j}} d \boldsymbol{r}^{\prime} \boldsymbol{b}_{n, j}^{R}\left(\boldsymbol{r}^{\prime}\right) \frac{\partial g\left(\boldsymbol{r}, \boldsymbol{r}^{\prime}\right)}{\partial n^{\prime}} \\
& +\int_{S_{m, i}} d \boldsymbol{r} \boldsymbol{t}_{m, i}^{R}(\boldsymbol{r}) \cdot \hat{\boldsymbol{n}}^{\prime} \int_{S_{n, j}} d \boldsymbol{r}^{\prime} \nabla^{\prime} \cdot\left[\boldsymbol{b}_{n, j}^{R}\left(\boldsymbol{r}^{\prime}\right) g\left(\boldsymbol{r}, \boldsymbol{r}^{\prime}\right)\right] \\
& -\int_{S_{m, i}} d \boldsymbol{r} \boldsymbol{t}_{m, i}^{R}(\boldsymbol{r}) \cdot \hat{\boldsymbol{n}}^{\prime} \int_{S_{n, j}} d \boldsymbol{r}^{\prime} g\left(\boldsymbol{r}, \boldsymbol{r}^{\prime}\right) \nabla^{\prime} \cdot \boldsymbol{b}_{n, j}^{R}\left(\boldsymbol{r}^{\prime}\right) \\
= & Z_{1}+Z_{2}+Z_{3}+Z_{4} .
\end{aligned}
$$

In (20), $Z_{4}$ can be further simplified since the RWG functions are divergence-conforming, having a constant charge distribution as

$$
\nabla \cdot \boldsymbol{f}^{R}(\boldsymbol{r})=\left\{\begin{array}{cc}
\frac{l}{A_{1}}, & r \in S_{1} \\
\frac{-l}{A_{2}}, & r \in S_{2} \\
0, & \text { otherwise }
\end{array}\right\}
$$
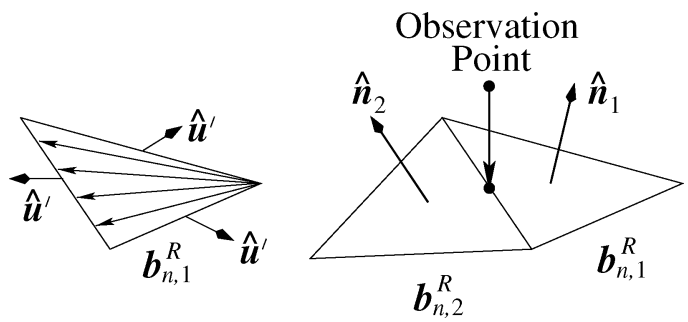

Fig. 5. (a) The half basis function $\boldsymbol{b}_{n, 1}^{R}$ with the outwardly perpendicular direction $\hat{\boldsymbol{u}}^{\prime}$ around the triangle. (b) Observation point approaching the edge of the basis function $\boldsymbol{b}_{n}^{R}=\boldsymbol{b}_{n, 1}^{R}+\boldsymbol{b}_{n, 2}^{R}$.

To evaluate $Z_{3}$ in (20) numerically, it is desirable to convert the inner integral on the surface to a line integral as

$$
\int_{S_{n, j}} d \boldsymbol{r}^{\prime} \nabla^{\prime} \cdot\left[\boldsymbol{b}_{n, j}^{R}\left(\boldsymbol{r}^{\prime}\right) g\left(\boldsymbol{r}, \boldsymbol{r}^{\prime}\right)\right]=\int_{\partial S_{n, j}} d \boldsymbol{r}^{\prime} \hat{\boldsymbol{u}}^{\prime} \cdot \boldsymbol{b}_{n, j}^{R}\left(\boldsymbol{r}^{\prime}\right) g\left(\boldsymbol{r}, \boldsymbol{r}^{\prime}\right)
$$

where $\partial S_{n, j}$ represents the contour around the basis triangle and $\hat{\boldsymbol{u}}^{\prime}$ is the unit vector directed perpendicularly outward the triangle as shown in Fig. 5(a). Considering the vector distribution of the RWG function, it is obvious that $\hat{\boldsymbol{u}}^{\prime} \cdot \boldsymbol{b}_{n, j}^{R}\left(\boldsymbol{r}^{\prime}\right)$ evaluates to zero on two of the edges since the function is defined parallel to these edges. On the other hand, for the edge $n$, on which the BF is defined, $\hat{\boldsymbol{u}}^{\prime} \cdot \boldsymbol{b}_{n, j}^{R}\left(\boldsymbol{r}^{\prime}\right)= \pm 1$ and (22) can be written as

$$
\int_{S_{n, j}} d \boldsymbol{r}^{\prime} \nabla^{\prime} \cdot\left[\boldsymbol{b}_{n, j}^{R}\left(\boldsymbol{r}^{\prime}\right) g\left(\boldsymbol{r}, \boldsymbol{r}^{\prime}\right)\right]= \pm \int_{\boldsymbol{l}_{n}} d \boldsymbol{r}^{\prime} g\left(\boldsymbol{r}, \boldsymbol{r}^{\prime}\right)
$$

which is simply the line integral of the Green's function along the edge $n$ and singular when the observation point approaches to this edge. This logarithmic singularity, occurring in the case of the near-neighbor (touching) interactions, has been extensively investigated for the RWG functions [11], [12], and the $\hat{\boldsymbol{n}} \times$ RWG functions. To observe this, Fig. 5(b) shows the RWG $\mathrm{BF} \boldsymbol{b}_{n}^{R}(\boldsymbol{r})$ and we note that

$$
\begin{aligned}
\int_{S_{n, 1}} d \boldsymbol{r}^{\prime} \nabla^{\prime} \cdot\left[\boldsymbol{b}_{n, 1}^{R}\left(\boldsymbol{r}^{\prime}\right) g\left(\boldsymbol{r}, \boldsymbol{r}^{\prime}\right)\right] & =\int_{l_{n}} d \boldsymbol{r}^{\prime} g\left(\boldsymbol{r}, \boldsymbol{r}^{\prime}\right) \\
\int_{S_{n, 2}} d \boldsymbol{r}^{\prime} \nabla^{\prime} \cdot\left[\boldsymbol{b}_{n, 2}^{R}\left(\boldsymbol{r}^{\prime}\right) g\left(\boldsymbol{r}, \boldsymbol{r}^{\prime}\right)\right] & =-\int_{l_{n}} d \boldsymbol{r}^{\prime} g\left(\boldsymbol{r}, \boldsymbol{r}^{\prime}\right) .
\end{aligned}
$$

It is encouraging to get exactly negative contributions from the two triangles sharing the $n$th edge, however, the annihilation of $Z_{3}$ in (20) occurs only when $\hat{\boldsymbol{n}}_{1}=\hat{\boldsymbol{n}}_{2}$, i.e., when the two triangles are on the same plane. When the BF is nonplanar, the singular results of (24) and (25), arising as the observation point approaches the common edge as shown in Fig. 5(b), do not cancel each other. As a result, the continuity of the parallel component of the $\hat{\boldsymbol{n}} \times$ RWG functions on the edge is not the remedy of the singularity problem [20], and the contribution of $Z_{3}$ cannot be discarded.

Despite the singularity of the integral in (23), numerical evaluation of $Z_{3}$ is not difficult since the singularity is logarithmic. For the near-neighbor interactions between the touching basis and testing triangles, sufficient number of observation points can be sampled inside the testing triangle to accurately compute the 
double integration. In addition to this, the order of the integrals can be exchanged as [21]

$$
\begin{aligned}
\int_{S_{m, i}} d \boldsymbol{r} \boldsymbol{t}_{m, i}^{R}(\boldsymbol{r}) \cdot \hat{\boldsymbol{n}}^{\prime} \int_{\partial S_{n, j}} d \boldsymbol{r}^{\prime} \hat{\boldsymbol{u}}^{\prime} \cdot \boldsymbol{b}_{n, j}^{R}\left(\boldsymbol{r}^{\prime}\right) g\left(\boldsymbol{r}, \boldsymbol{r}^{\prime}\right) \\
=\int_{\partial S_{n, j}} d \boldsymbol{r}^{\prime} \hat{\boldsymbol{u}}^{\prime} \cdot \boldsymbol{b}_{n, j}^{R}\left(\boldsymbol{r}^{\prime}\right) \hat{\boldsymbol{n}}^{\prime} \cdot \int_{S_{m, i}} d \boldsymbol{r} \boldsymbol{t}_{m, i}^{R}(\boldsymbol{r}) g\left(\boldsymbol{r}, \boldsymbol{r}^{\prime}\right) \\
= \pm \int_{l_{n}} d \boldsymbol{r}^{\prime} \hat{\boldsymbol{n}}^{\prime} \cdot \int_{S_{m, i}} d \boldsymbol{r} \boldsymbol{t}_{m, i}^{R}(\boldsymbol{r}) g\left(\boldsymbol{r}, \boldsymbol{r}^{\prime}\right)
\end{aligned}
$$

where the inner integral is now bounded for every case including the sampling of the testing points on the edge of the basis triangle.

There is also a formulation for the RWG functions that is analogous to the alternative formulation for the $\hat{\boldsymbol{n}} \times$ RWG functions presented in this section. To observe this, we manipulate the expression in (20) as

$$
\begin{aligned}
Z_{m n, i j}^{\mathrm{nRWG}}= & \int_{S_{m, i}} d \boldsymbol{r} \boldsymbol{t}_{m, i}^{R}(\boldsymbol{r}) \cdot \boldsymbol{b}_{n, j}^{R}(\boldsymbol{r}) \\
& -\int_{S_{n, j}} d \boldsymbol{r}^{\prime} \boldsymbol{b}_{n, j}^{R}\left(\boldsymbol{r}^{\prime}\right) \cdot \int_{S_{m, i}} d \boldsymbol{r t}_{m, i}^{R}(\boldsymbol{r}) \frac{\partial g\left(\boldsymbol{r}, \boldsymbol{r}^{\prime}\right)}{\partial n^{\prime}} \\
& +\int_{S_{n, j}} d \boldsymbol{r}^{\prime} \nabla^{\prime} \cdot\left[\boldsymbol{b}_{n, j}^{R}\left(\boldsymbol{r}^{\prime}\right) \hat{\boldsymbol{n}}^{\prime} \cdot \int_{S_{m, i}} d \boldsymbol{r} t_{m, i}^{R}(\boldsymbol{r}) g\left(\boldsymbol{r}, \boldsymbol{r}^{\prime}\right)\right] \\
& -\int_{S_{n, j}} d \boldsymbol{r}^{\prime} \nabla^{\prime} \cdot \boldsymbol{b}_{n, j}^{R}\left(\boldsymbol{r}^{\prime}\right) \hat{\boldsymbol{n}}^{\prime} \cdot \int_{S_{m, i}} d \boldsymbol{r} \boldsymbol{t}_{m, i}^{R}(\boldsymbol{r}) g\left(\boldsymbol{r}, \boldsymbol{r}^{\prime}\right)(27)
\end{aligned}
$$

which is related to

$$
\begin{aligned}
Z_{m n, i j}^{\mathrm{RWG}}= & \int_{S_{m, i}} d \boldsymbol{r} \boldsymbol{t}_{m, i}^{R}(\boldsymbol{r}) \cdot \boldsymbol{b}_{n, j}^{R}(\boldsymbol{r}) \\
& +\int_{S_{m, i}} d \boldsymbol{r} \boldsymbol{t}_{m, i}^{R}(\boldsymbol{r}) \cdot \int_{S_{n, j}} d \boldsymbol{r}^{\prime} \boldsymbol{b}_{n, j}^{R}\left(\boldsymbol{r}^{\prime}\right) \frac{\partial g\left(\boldsymbol{r}, \boldsymbol{r}^{\prime}\right)}{\partial n} \\
& -\int_{S_{m, i}} d \boldsymbol{r} \nabla \cdot\left[\boldsymbol{t}_{m, i}^{R}(\boldsymbol{r}) \hat{\boldsymbol{n}} \cdot \int_{S_{n, j}} d \boldsymbol{r}^{\prime} \boldsymbol{b}_{n, j}^{R}\left(\boldsymbol{r}^{\prime}\right) g\left(\boldsymbol{r}, \boldsymbol{r}^{\prime}\right)\right] \\
& +\int_{S_{m, i}} d \boldsymbol{r} \nabla \cdot \boldsymbol{t}_{m, i}^{R}(\boldsymbol{r}) \hat{\boldsymbol{n}} \cdot \int_{S_{n, j}} d \boldsymbol{r}^{\prime} \boldsymbol{b}_{n, j}^{R}\left(\boldsymbol{r}^{\prime}\right) g\left(\boldsymbol{r}, \boldsymbol{r}^{\prime}\right)
\end{aligned}
$$

according to (14) and (16). The formulation in (28) is proposed in [12] as the "third formulation" to calculate the interactions of the RWG functions in the MFIE.

\section{RESULTS}

To compare the accuracy of the solutions obtained by using the RWG and the $\hat{\boldsymbol{n}} \times$ RWG functions, the first example is a scattering problem of a perfectly conducting sphere of radius $a=\lambda$ and $2 \lambda$. Figs. 6 and 7 demonstrate the normalized backscattered and forward-scattered $\mathrm{RCS} / \pi a^{2}$ values, respectively, obtained by the FMM implementations employing the RWG and the $\hat{\boldsymbol{n}} \times$ RWG functions. The problem is solved for

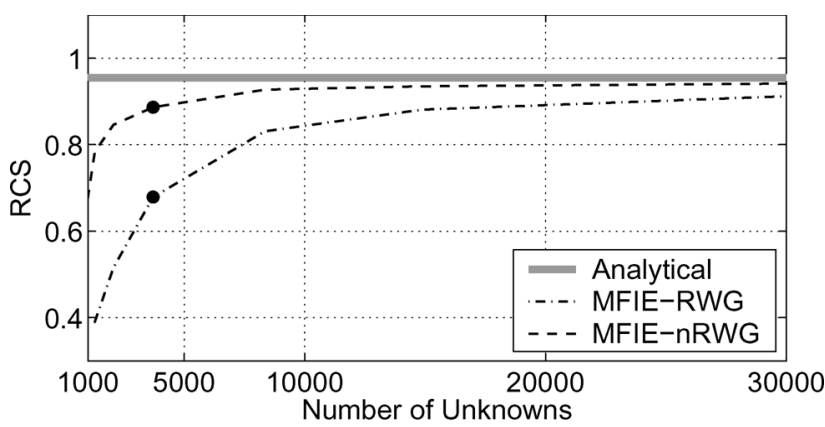

(a)

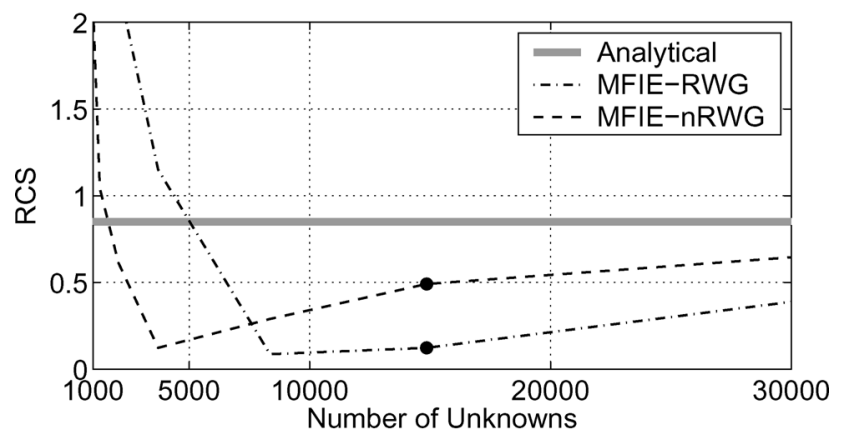

(b)

Fig. 6. Normalized backscattered RCS (RCS $/ \pi a^{2}$, dimensionless) of the perfectly conducting sphere of radius (a) $a=\lambda$ and (b) $a=2 \lambda$ with respect to the number of unknowns. The dots on the curves correspond to the $\lambda / 10$ discretization.

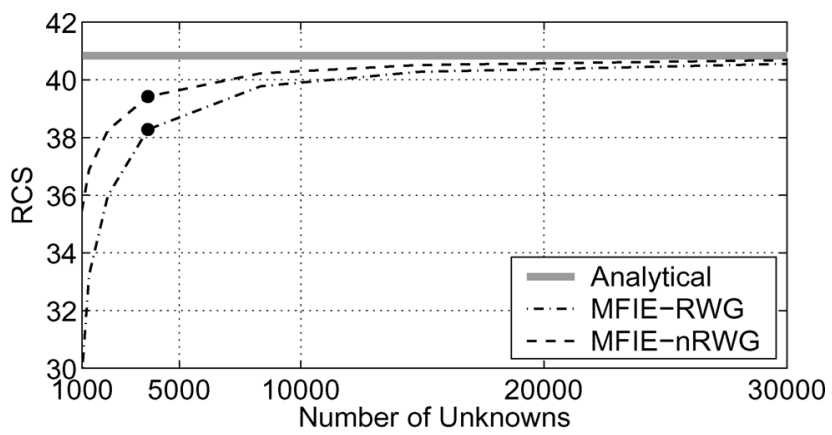

(a)

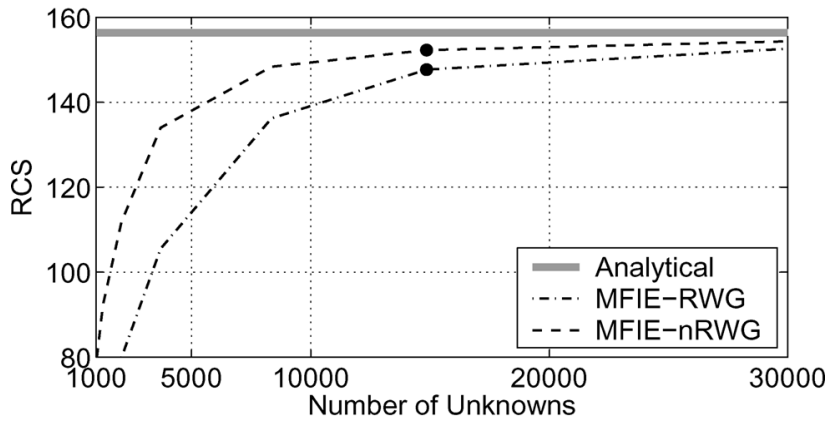

(b)

Fig. 7. Normalized forward-scattered RCS (RCS $/ \pi a^{2}$, dimensionless) of the perfectly conducting sphere of radius (a) $a=\lambda$ and (b) $a=2 \lambda$ with respect to the number of unknowns. The dots on the curves correspond to the $\lambda / 10$ discretization.

various triangulations involving approximately 1000 to 30,000 unknowns $(N)$. In both figures, we observe that, as $N$ increases, RCS values obtained with the $\hat{\boldsymbol{n}} \times$ RWG functions converge 


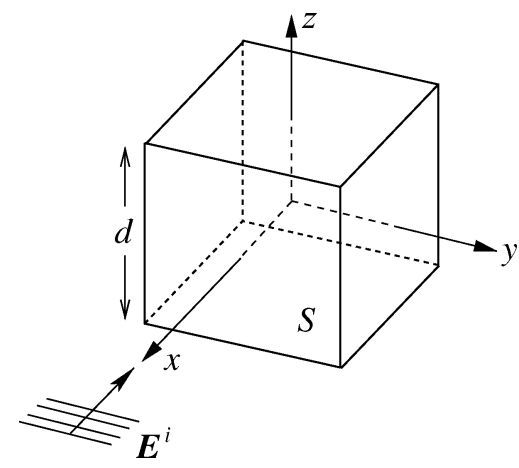

Fig. 8. Perfectly conducting cube with edges of $d$.

to the exact results (i.e., analytical Mie-series solutions) faster than those obtained with the RWG functions, i.e., RCS values obtained with the $\hat{\boldsymbol{n}} \times$ RWG functions are more accurate for all discretization densities. The improvement obtained with the $\hat{\boldsymbol{n}} \times$ RWG functions over the RWG functions is significant even for the $\lambda / 10$ discretization, which is commonly used as a rule of thumb in numerical solutions.

The second example involves a perfectly conducting cube with edges of $d=1 \mathrm{~m}$ as shown in Fig. 8, illuminated by a plane wave propagating in the $-x$ direction with the polarization of the electric field in the $y$ direction. The problem is solved for $d=\lambda$ and $2 \lambda$ with various triangulations from $20 \mathrm{~cm}$ to 3 $\mathrm{cm}$, corresponding to approximately 500 to 18,000 unknowns, respectively. Figs. 9 and 10 show the magnitude of the $y$ component of the normalized surface current $\left(J_{y} / H^{i}\right.$, dimensionless) induced on the front surface of the cube (at $x=0.5 \mathrm{~m}$ ). In Fig. 9, where $d=\lambda$ and the triangulation size is about $\lambda / 10$, and in Fig. 10, where $d=2 \lambda$ and the triangulation size is about $\lambda / 12.5$, we observe that the modelling of the surface current is different for the EFIE and the MFIE implementations using the RWG BFs. Even though the plots look similar, significant differences can be noticed by focusing on the calculated values at $z=$ $\pm 0.5 \mathrm{~m}$, where the induced current is expected to be singular. The combination of the geometry discretization and the RWG BFs does not allow the computed current values to become singular at those two edges of the cube. Since the physically correct solution is singular, the more accurate the computed solutions get, the higher values they would assume at those edges in order to match the singularity as closely as possible. Comparing Fig. 9(a) to (b) and Fig. 10(a) to (b), EFIE solutions are seen to have consistently higher values than the MFIE solutions in spite of employing exactly the same discretization and the RWG BFs. Since the only difference is in the integral-equation formulation, this comparison demonstrates the inaccuracy of the MFIE relative to the EFIE.

Figs. 9(c) and 10(c) show that, using the $\hat{\boldsymbol{n}} \times$ RWG functions, the modelling of the surface current is visibly improved compared to the RWG functions, especially in the representation of the singular currents flowing along the edges. We observe that the use of the $\hat{\boldsymbol{n}} \times$ RWG functions in the MFIE solution provides higher values at $z= \pm 0.5 \mathrm{~m}$ than the use of the RWG functions with either the EFIE or the MFIE. It should be remarked that this is merely an indicator for the improved accuracy of the

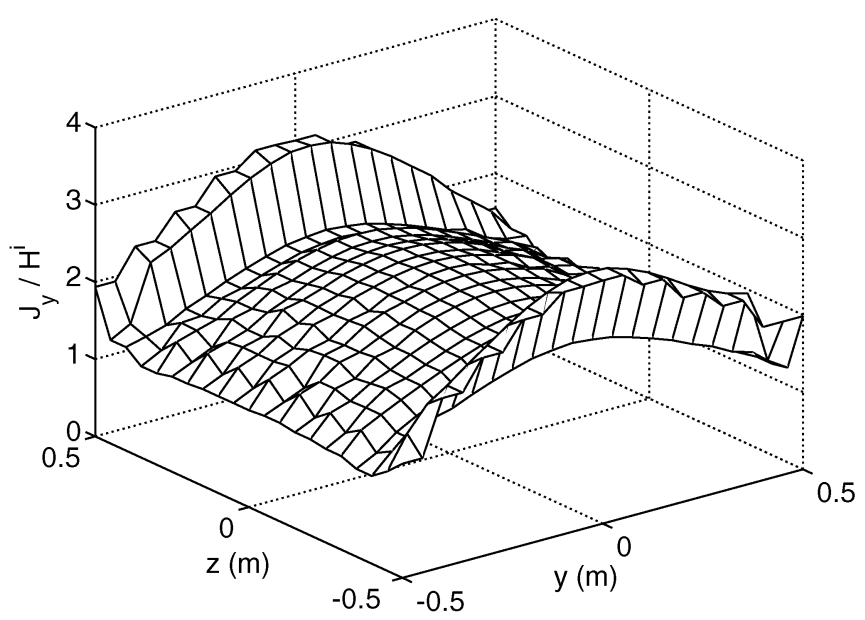

(a)

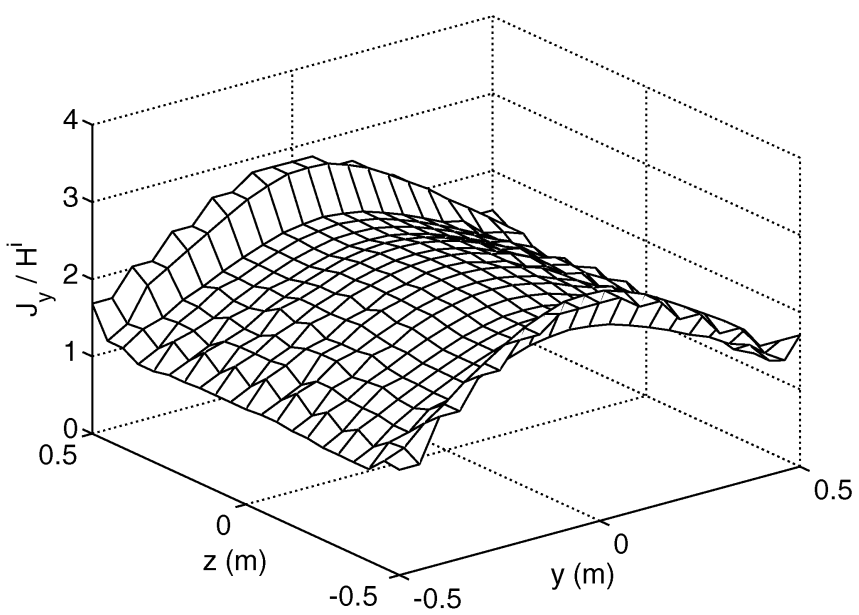

(b)

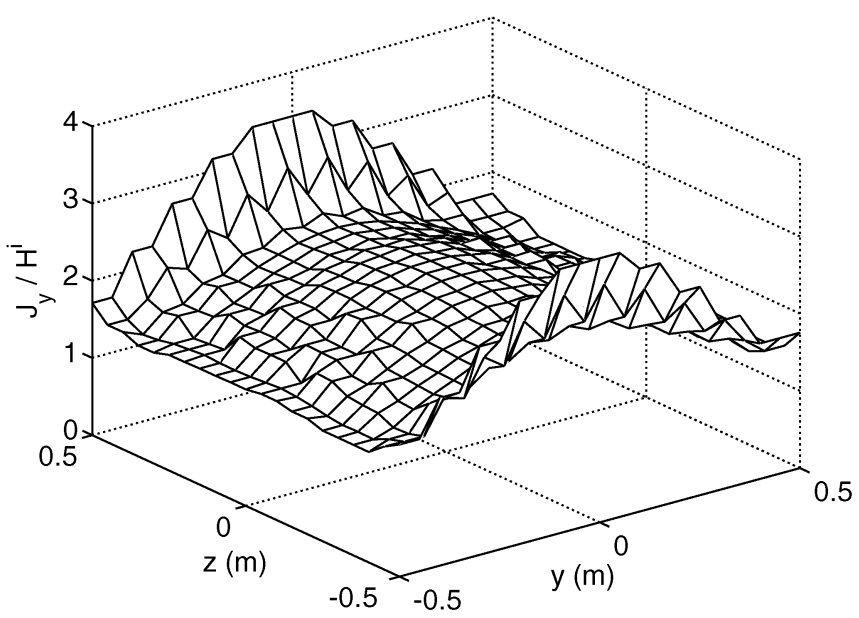

(c)

Fig. 9. Magnitude of the $y$ component of the normalized surface current $\left(\left|J_{y} / H^{i}\right|\right)$ induced on the front surface (at $x=0.5 \mathrm{~m}$ ) of the perfectly conducting cube in Fig. 8 with $d=\lambda$ and triangulation size of about $\lambda / 10$, obtained by using (a) EFIE with RWG, (b) MFIE with RWG, and (c) MFIE with $\hat{n} \times$ RWG.

MFIE with the use of the $\hat{\boldsymbol{n}} \times$ RWG functions, but not an absolute proof since the exact current distribution is not available for comparisons. Nevertheless, the RCS results are also supporting the inferences obtained from the current solutions. 


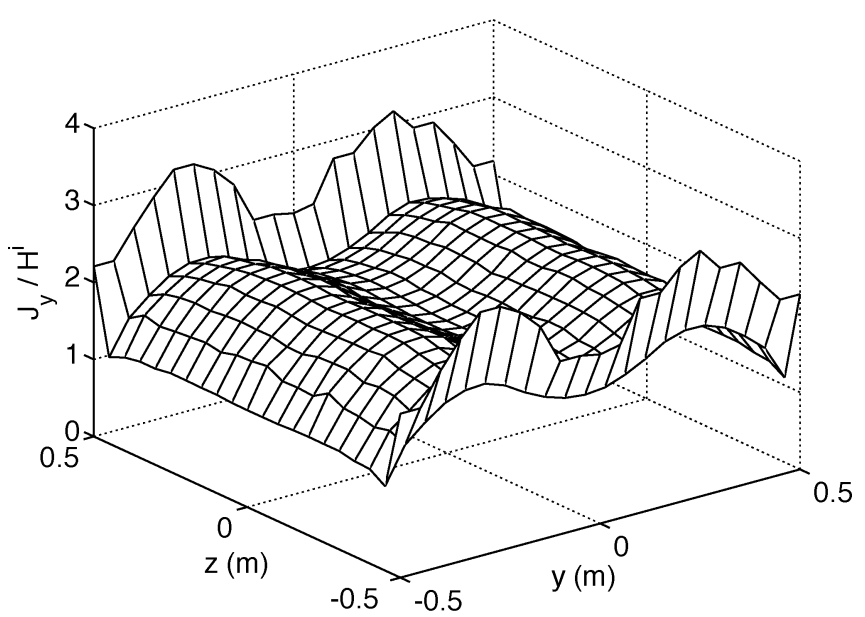

(a)

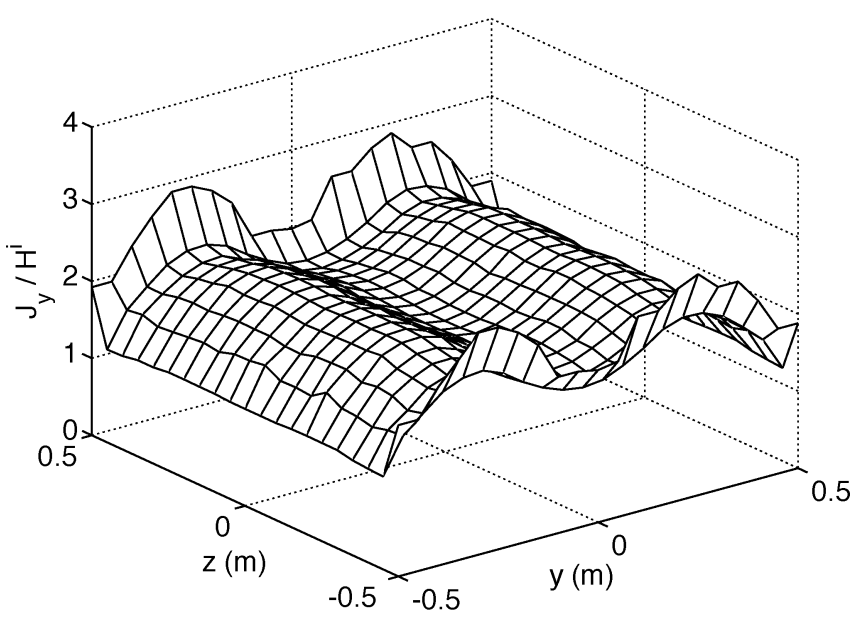

(b)

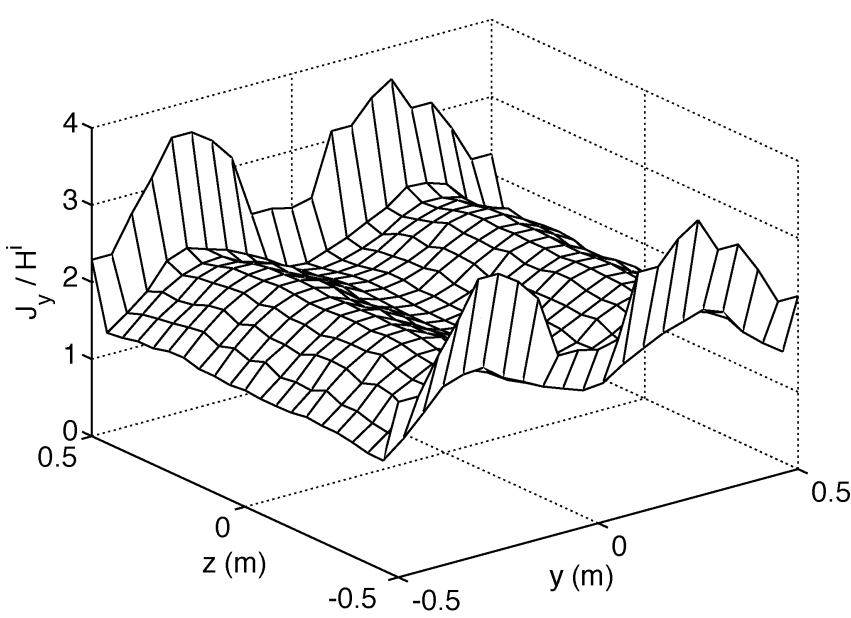

(c)

Fig. 10. Magnitude of the $y$ component of the normalized surface current $\left(\left|J_{y} / H^{i}\right|\right)$ induced on the front surface (at $x=0.5 \mathrm{~m}$ ) of the perfectly conducting cube in Fig. 8 with $d=2 \lambda$ and triangulation size about $\lambda / 12.5$, obtained by using (a) EFIE with RWG, (b) MFIE with RWG, and (c) MFIE with $\hat{n} \times$ RWG.

Finally, Figs. 11 and 12 demonstrate the backscattered and forward-scattered RCS values (in $\mathrm{m}^{2}$ ), respectively, for two different frequencies corresponding to $d=\lambda$ and $2 \lambda$. Comparing the MFIE results, we observe faster convergence (with respect to the improved discretization and the increasing $N$ ) of the RCS

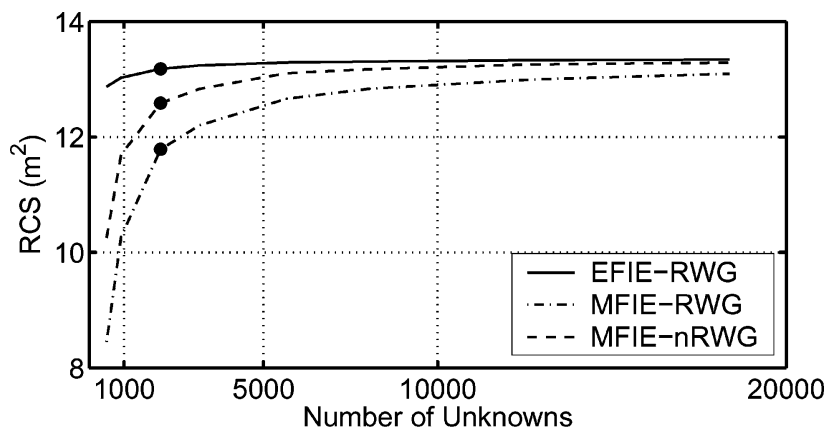

(a)

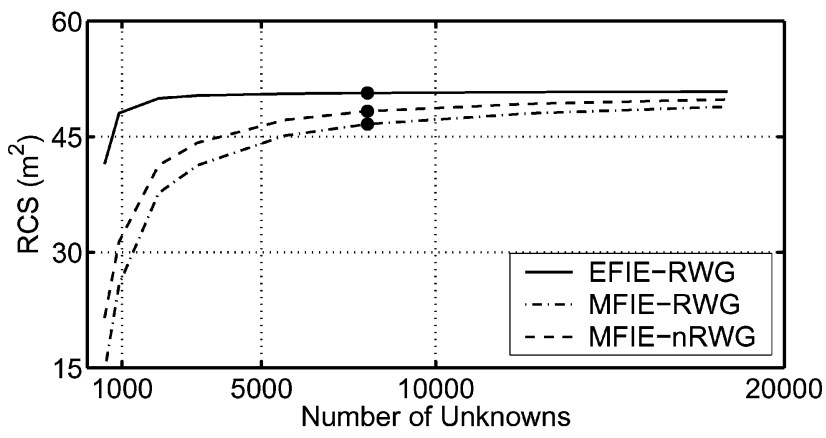

(b)

Fig. 11. Backscattered RCS $\left(\mathrm{m}^{2}\right)$ of the perfectly conducting cubes with edges of (a) $\lambda$ and (b) $2 \lambda$ with respect to the number of unknowns. The dots on the curves correspond to the $\lambda / 10$ discretization.

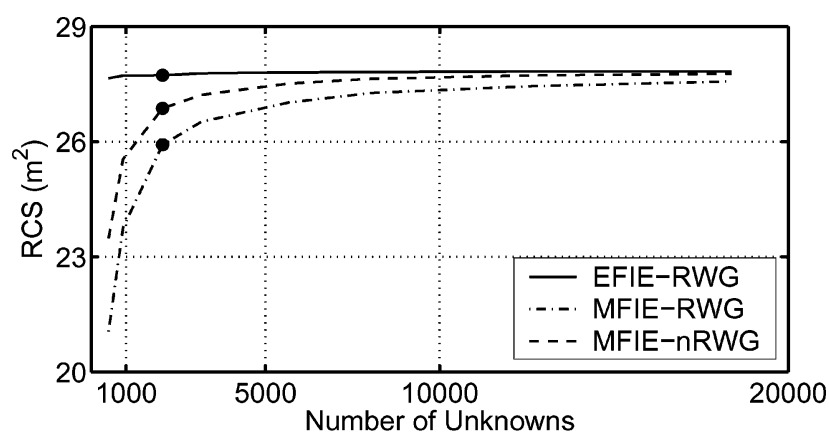

(a)

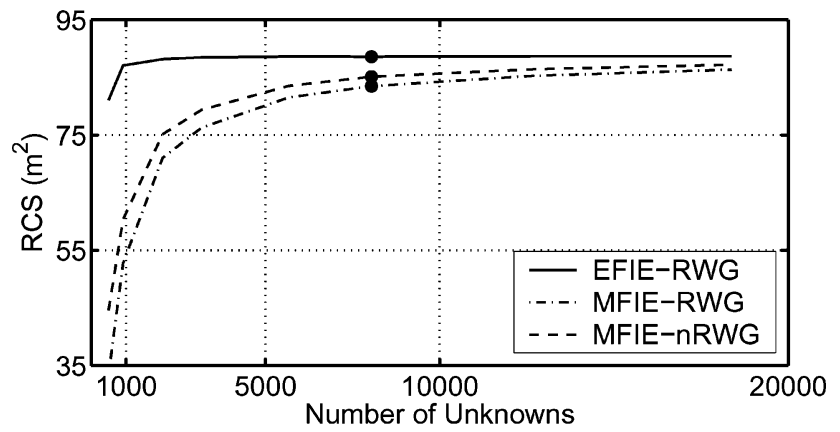

(b)

Fig. 12. Forward-scattered RCS $\left(\mathrm{m}^{2}\right)$ of the perfectly conducting cubes with edges of (a) $\lambda$ and (b) $2 \lambda$ with respect to the number of unknowns. The dots on the curves correspond to the $\lambda / 10$ discretization.

values with the $\hat{\boldsymbol{n}} \times$ RWG functions. The improvement is significant especially for relatively coarse discretizations, even for the commonly used $\lambda / 10$ triangulation, as marked on the curves. 


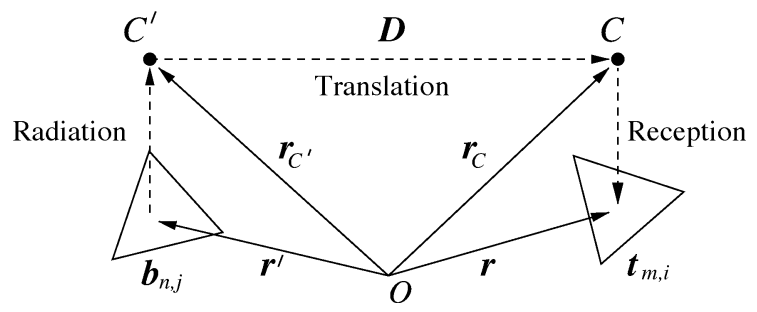

Fig. 13. Calculation of the interactions in the FMM.

\section{CONCLUSION}

In this paper, we consider a novel implementation of the MFIE employing the curl-conforming $\hat{\boldsymbol{n}} \times$ RWG BTFs for improved current modelling. Both required formulations and numerical schemes for the accurate evaluation thereof in the context of the MOM and the FMM are outlined. The results presented for the sphere and cube problems demonstrate the significant improvement in the accuracy of the solutions obtained with the $\hat{\boldsymbol{n}} \times$ RWG functions instead of the commonly used RWG functions. In the light of the lessons learned by RWG functions with the $\hat{\boldsymbol{n}} \times$ RWG functions in this paper, further improvements in the accuracy of the MFIE can be obtained with other BFs resulting in better current modelling. This will be reported elsewhere [27].

\section{APPENDIX}

\section{CALCULATION OF THE INTERACTIONS IN THE FMM}

In the FMM and the MLFMA, far-field interactions of the MFIE are calculated as [7]

$$
Z_{m n, i j}=\left(\frac{i k}{4 \pi}\right)^{2} \int d^{2} \hat{\boldsymbol{k}} \boldsymbol{F}_{m, i}^{C}(\hat{\boldsymbol{k}}) T_{L}(k,|\boldsymbol{D}|, \hat{\boldsymbol{D}} \cdot \hat{\boldsymbol{k}}) \cdot \boldsymbol{F}_{n, j}^{C^{\prime}}(\hat{\boldsymbol{k}})
$$

where $T_{L}(k,|D|, \hat{\boldsymbol{D}} \cdot \hat{k})$ is the translation function expressed as

$$
T_{L}(k,|\boldsymbol{D}| \hat{\boldsymbol{D}} \cdot \hat{\boldsymbol{k}})=\sum_{l=0}^{L} i^{l}(2 l+1) h_{l}^{(1)}(k D) P_{l}(\hat{\boldsymbol{D}} \cdot \hat{\boldsymbol{k}})
$$

in terms of the spherical Hankel function of the first kind $h_{l}^{(1)}$ and the Legendre polynomial $P_{l}$. Evaluation of (29) is illustrated in Fig. 13, where the radiation of the half BF $\boldsymbol{b}_{n, j}(\boldsymbol{r})$ with respect to a near point $C^{\prime}$ located at $\boldsymbol{r}_{C^{\prime}}$

$$
\boldsymbol{F}_{n, j}^{C^{\prime}}(\hat{k})=\int_{S_{n, j}} d \boldsymbol{r}^{\prime} e^{-i \boldsymbol{k} \cdot\left(\boldsymbol{r}^{\prime}-\boldsymbol{r}_{C^{\prime}}\right)} \boldsymbol{b}_{n, j}\left(\boldsymbol{r}^{\prime}\right)
$$

is translated by $T_{L}(k,|\boldsymbol{D}|, \hat{\boldsymbol{D}} \cdot \hat{k})$ into an incoming wave at point $C$ located at $\boldsymbol{r}_{C}=\boldsymbol{r}_{C^{\prime}}+\boldsymbol{D}$, and then received by the half $\mathrm{TF} \boldsymbol{t}_{m, i}(\boldsymbol{r})$ that has a receiving pattern with respect to the close point $C$ as

$$
\boldsymbol{F}_{m, i}^{C}(\hat{\boldsymbol{k}})=-\hat{\boldsymbol{k}} \times \int_{S_{m, i}} d \boldsymbol{r} e^{i \boldsymbol{k} \cdot\left(\boldsymbol{r}-\boldsymbol{r}_{C}\right)} \boldsymbol{t}_{m, i}(\boldsymbol{r}) \times \hat{\boldsymbol{n}} .
$$

The translation function in (30) is independent of the BTFs; only the radiation pattern in (31) and the receiving pattern in
(32) need to be modified for different choices of the BTFs. For $\hat{\boldsymbol{n}} \times$ RWG BFs, (31) can be evaluated as

$$
\begin{aligned}
\boldsymbol{F}_{n, j}^{C^{\prime}}(\hat{\boldsymbol{k}}) & =\hat{\boldsymbol{n}}^{\prime} \times \int_{S_{n, j}} d \boldsymbol{r}^{\prime} e^{-i \boldsymbol{k} \cdot\left(\boldsymbol{r}^{\prime}-\boldsymbol{r}_{C^{\prime}}\right)} \boldsymbol{b}_{n, j}^{R}\left(\boldsymbol{r}^{\prime}\right) \\
& = \pm \frac{l_{n}}{2 A_{n, j}} \hat{\boldsymbol{n}}^{\prime} \times \int_{S_{n, j}} d \boldsymbol{r}^{\prime} e^{-i \boldsymbol{k} \cdot\left(\boldsymbol{r}^{\prime}-\boldsymbol{r}_{C^{\prime}}\right)}\left(\boldsymbol{r}^{\prime}-\boldsymbol{r}_{n, j}\right)
\end{aligned}
$$

using the definitions in (10) and (11), and extracting the constant normal outside the integral. Similarly, (32) can be written for the $\hat{\boldsymbol{n}} \times$ RWG TFs as

$$
\begin{aligned}
\boldsymbol{F}_{m, i}^{C}(\hat{\boldsymbol{k}}) & =-\hat{\boldsymbol{k}} \times \int_{S_{m, i}} d \boldsymbol{r} e^{i \boldsymbol{k} \cdot\left(\boldsymbol{r}-\boldsymbol{r}_{C}\right)} \boldsymbol{t}_{m, i}^{R}(\boldsymbol{r}) \\
& =\mp \frac{l_{n}}{2 A_{n, j}} \hat{\boldsymbol{k}} \times \int_{S_{m, i}} d \boldsymbol{r} e^{i \boldsymbol{k} \cdot\left(\boldsymbol{r}-\boldsymbol{r}_{C}\right)}\left(\boldsymbol{r}-\boldsymbol{r}_{m, i}\right),
\end{aligned}
$$

where the integral is simply the complex conjugate of the integral in (33). The integrals in (33) and (34) can be evaluated analytically without resorting to any numerical quadrature.

\section{REFERENCES}

[1] A. J. Poggio and E. K. Miller, "Integral equation solutions of three-dimensional scattering problems," in Computer Techniques for Electromagnetics, R. Mittra, Ed. Oxford, U.K.: Permagon Press, 1973, ch. 4.

[2] J. R. Mautz and R. F. Harrington, "H-field, E-field, and combined field solutions for conducting bodies of revolution," $A E \ddot{U}$, vol. 32, no. 4, pp. 157-164, Apr. 1978.

[3] N. Morita, N. Kumagai, and J. R. Mautz, Integral Equation Methods for Electromagnetics. Boston, MA: Artech House, 1990.

[4] Y. Zhang, T. J. Cui, W. C. Chew, and J.-S. Zhao, "Magnetic field integral equation at very low frequencies," IEEE Trans. Antennas Propag., vol. AP-51, no. 8, pp. 1864-1871, Aug. 2003.

[5] R. F. Harrington, Field Computation by Moment Methods. New York: Macmillan, 1968.

[6] R. Coifman, V. Rokhlin, and S. Wandzura, "The fast multipole method for the wave equation: a pedestrian prescription," IEEE Antennas Propag. Mag., vol. 35, no. 3, pp. 7-12, Jun. 1993.

[7] W. C. Chew, J.-M. Jin, E. Michielssen, and J. Song, Fast and Efficient Algorithms in Computational Electromagnetics. Boston, MA: Artech House, 2001.

[8] C.-C. Lu and W. C. Chew, "Multilevel fast multipole algorithm for electromagnetic scattering by large complex objects," IEEE Trans. Antennas Propag., vol. 45, no. 10, pp. 1488-1493, Oct. 1997.

[9] S. M. Rao, D. R. Wilton, and A. W. Glisson, "Electromagnetic scattering by surfaces of arbitrary shape," IEEE Trans. Antennas Propag., vol. AP-30, no. 3, pp. 409-418, May 1982.

[10] J. M. Rius, E. Úbeda, and J. Parrón, "On the testing of the magnetic field integral equation with RWG basis functions in method of moments," IEEE Trans. Antennas Propag., vol. 49, pp. 1550-1553, Nov. 2001.

[11] Ö. Ergül and L. Gürel, "Investigation of the inaccuracy of the MFIE discretized with the RWG basis functions," in Proc. IEEE AP-S Int. Symp., Monterey, CA, Jun. 2004, pp. 3393-3396.

[12] L. Gürel and Ö. Ergül, "Singularity of the magnetic-field integral equation and its extraction," IEEE Antennas Wireless Propag. Lett., vol. 4, pp. 229-232, 2005.

[13] Ö. Ergül and L. Gürel, "Improved testing of the magnetic-field integral equation," IEEE Microwave Wireless Comp. Lett., vol. 15, no. 10, pp. 615-617, Oct. 2005.

[14] _ , "Solid-angle factor in the magnetic-field integral equation," $M i$ crowave Opt. Technol. Lett., vol. 45, no. 5, pp. 452-456, Jun. 2005.

[15] Ö. Ergül and L. Gürel, "Improving the accuracy of the MFIE with the choice of basis functions," in Proc. IEEE AP-S Int. Symp., Monterey, CA, Jun. 2004, pp. 3389-3392.

[16] S. M. Rao and D. R. Wilton, "E-field, H-field, and combined field solution for arbitrary shaped three-dimensional dielectric bodies," Electromagn., vol. 10, no. 4, pp. 407-421, 1990. 
[17] X.-Q. Sheng, J.-M. Jin, J. Song, W. C. Chew, and C.-C. Lu, "Solution of combined-field integral equation using multilevel fast multipole algorithm for scattering by homogeneous bodies," IEEE Trans. Antennas Propag., vol. 46, no. 11, pp. 1718-1726, Nov. 1998.

[18] A. F. Peterson, S. L. Ray, and R. Mittra, Computational Methods for Electromagnetics. New York: IEEE Press, 1998.

[19] E. Úbeda and J. M. Rius, "Curl-conforming MFIE in the analysis of perfectly conducting sharply-edged objects," in Proc. IEEE AP-S Int. Symp., Monterey, CA, Jun. 2004, pp. 4052-4055.

[20] A. F. Peterson, "Solution of the MFIE using curl-conforming basis functions," in Proc. IEEE AP-S Int. Symp., Atlanta, GA, Jun. 2002, vol. 1, pp. 70-73.

[21] P. Y. Oijala and M. Taskinen, "Calculation of CFIE impedance matrix elements with RWG and $\hat{\boldsymbol{n}} \times$ RWG functions," IEEE Trans. Antennas Propag., vol. 51, no. 8, pp. 1837-1846, Aug. 2003.

[22] L. Gürel and Ö. Ergül, "Comparisons of FMM implementations employing different formulations and iterative solvers," in Proc. IEEE AP-S Int. Symp., Columbus, OH, Jun. 2003, vol. 1, pp. 19-22.

[23] R. E. Hodges and Y. Rahmat-Samii, "The evaluation of MFIE integrals with the use of vector triangle basis functions," Microwave Opt. Technol. Lett., vol. 14, no. 1, pp. 9-14, Jan. 1997.

[24] D. R. Wilton, S. M. Rao, A. W. Glisson, D. H. Schaubert, O. M. Al-Bundak, and C. M. Butler, "Potential integrals for uniform and linear source distributions on polygonal and polyhedral domains," IEEE Trans. Antennas Propag., vol. AP-32, pp. 276-281, Mar. 1984.

[25] R. D. Graglia, "On the numerical integration of the linear shape functions times the 3-D Green's function or its gradient on a plane triangle," IEEE Trans. Antennas Propag., vol. 41, no. 10, pp. 1448-1455, Oct. 1993.

[26] L. Gürel, K. Sertel, and İ. K. Şendur, "On the choice of basis functions to model surface electric current densities in computational electromagnetics," Radio Sci., vol. 34, no. 6, pp. 1373-1387, Nov.-Dec. 1999.

[27] Ö. Ergül and L. Gürel, "Improving the accuracy of the magnetic-field integral equation with the linear-linear basis functions," Radio Sci., to appear 2006.

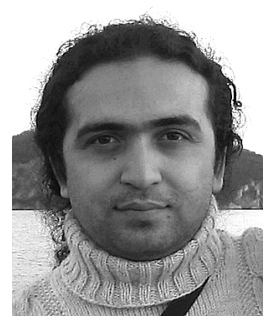

Özgür Ergül (S'98) was born in Yozgat, Turkey, 1978. He received the B.S. and M.S. degrees in electrical and electronics engineering from Bilkent University, Ankara, in 2001 and 2003, respectively. $\mathrm{He}$ is currently working toward the Ph.D. degree at Bilkent University.

From 2000 to 2005, he was affiliated with the Computational Electromagnetics Group at Bilkent University. Since 2001, he has served as a Teaching and Research Assistant in the Department of Electrical and Electronics Engineering at Bilkent University, where, since 2005, he has also been affiliated with the Computational Electromagnetics Research Center (BiLCEM). His research interests include fast and accurate algorithms for the solution of large and complicated structures, parallel programming, and iterative techniques.

Mr. Ergül's academic endeavors are supported by the Scientific and Technical Research Council of Turkey (TUBITAK) in the framework of a national Ph.D. scholarship.

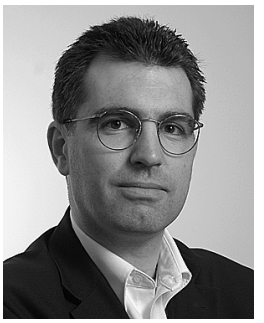

Levent Gürel (S'87-M'92-SM'97) received the B.Sc. degree from the Middle East Technical University (METU), Ankara, Turkey, in 1986, and the M.S. and $\mathrm{Ph} . \mathrm{D}$. degrees from the University of Illinois at Urbana-Champaign (UIUC), in 1988 and 1991, respectively, all in electrical engineering.

He joined the Thomas J. Watson Research Center of the International Business Machines Corporation, Yorktown Heights, New York, in 1991, where he worked as a Research Staff Member on the electromagnetic compatibility (EMC) problems related to electronic packaging, on the use of microwave processes in the manufacturing and testing of electronic circuits, and on the development of fast solvers for interconnect modeling. Since 1994, he has been a faculty member in the Department of Electrical and Electronics Engineering of the Bilkent University, Ankara, where he is currently a Professor. He was a Visiting Associate Professor at the Center for Computational Electromagnetics (CCEM) of the UIUC for one semester in 1997. He returned to the UIUC as a Visiting Professor in 2003-2005, and as an Adjunct Professor in 2005-2006. He founded the Computational Electromagnetics Research Center (BiLCEM) at Bilkent University in 2005, where he is serving as the Director. His research interests include the development of fast algorithms for computational electromagnetics (CEM) and the application thereof to scattering and radiation problems involving large and complicated scatterers, antennas and radars, frequency-selective surfaces, high-speed electronic circuits, optical and imaging systems, nanostructures, and metamaterials. He is also interested in the theoretical and computational aspects of electromagnetic compatibility and interference analyses. Ground penetrating radars and other subsurface scattering applications are also among his research interests.

Dr. Gürel is a member of the General Assembly of the European Microwave Association, a member of the USNC of the International Union of Radio Science (URSI), and the chairman of Commission E (Electromagnetic Noise and Interference) of URSI Turkey National Committee. He received two prestigious awards from the Turkish Academy of Sciences (TUBA) in 2002 and the Scientific and Technical Research Council of Turkey (TUBITAK) in 2003. He served as the Chairman of the AP/MTT/ED/EMC Chapter of the IEEE Turkey Section in 2000-2003. He founded the EMC Chapter in Turkey in 2000. He served as the cochairman of the 2003 IEEE International Symposium on Electromagnetic Compatibility. He is currently serving as an Associate Editor of Radio Science. 